\title{
The mediating effect of psychological resilience on the level of mindfulness and general well-being in patients with inflammatory bowel disease
}

\author{
Meifeng Wang", Xi Lu", Meihong Liu \\ Department of Gastroenterology, The First Affiliated Hospital of Nanjing Medical University (Jiangsu Provincial People's Hospital), Nanjing, China \\ Contributions: (I) Conception and design: M Liu; (II) Administrative support: M Liu; (III) Provision of study materials or patients: X Lu; (IV) \\ Collection and assembly of data: X Lu; (V) Data analysis and interpretation: M Wang; (VI) Manuscript writing: All authors; (VII) Final approval of \\ manuscript: All authors. \\ \#The authors contributed equally to this work. \\ Correspondence to: Meihong Liu. Department of Gastroenterology, The First Affiliated Hospital of Nanjing Medical University (Jiangsu Provincial \\ People's Hospital), 300 Guangzhou Road, Nanjing 210029, China. Email: 67496437@qq.com.
}

Background: The incidence of inflammatory bowel disease (IBD) increases annually, and IBD patients
may experience various psychological symptoms. People with higher levels of psychological resilience tend
to cope with negative events in a positive way. So here we explored the correlation of mindfulness level,
psychological resilience, and general well-being in patients with inflammatory bowel disease (IBD), and the
mediating effect of psychological resilience on mindfulness level and happiness in IBD.
Methods: Five Facet Mindfulness Questionnaire (FFMQ), Connor-Davidson Resilience Scale (CD-
RICS), and General Well-Being Schedule (GWB) were used for investigation among the randomly selected
IBD patients. Pearson correlation analysis and the structural equation model by Amos 24.0 were applied to
explore the relationship between mindfulness level, psychological resilience, and general well-being. The
mediating effect of psychological resilience was detected by bootstrap method.

Results: A total of 159 of the 184 distributed questionnaires were recovered (recovery rate $73.3 \%$ ), and 135 of them were used for subsequent investigations. In IBD patients, the score of mindfulness level, psychological resilience, and general well-being were $(119.95 \pm 8.64),(58.37 \pm 19.32)$, and $(73.59 \pm 13.88)$, respectively. Both male and female score were lower than ordinary model (52.7\% and 42.9\%, respectively). Pearson results displayed that mindfulness level $(\mathrm{r}=0.312-0.390, \mathrm{P}<0.01)$ and overall well-being $(\mathrm{r}=0.490-$ $0.590, \mathrm{P}<0.01)$ were positively related to psychological resilience and its 3 dimensions, respectively. Similarly, mindfulness level was positively correlated with overall well-being $(r=0.391, P<0.01)$. Mediating effect of psychological resilience between mindfulness level and overall well-being was $0.232(\mathrm{P}=0.001)$. The $95 \%$ confidence interval $(95 \% \mathrm{CI})$ of psychological resilience as mediating effect $(59.3 \%$ of total) was (0.138 to 0.332$)$.

Conclusions: We concluded that psychological resilience in IBD patients has a partially mediating effect between mindfulness level and overall well-being.

Keywords: Psychological resilience; inflammatory bowel disease; mindfulness; overall well-being

Submitted Jun 29, 2021. Accepted for publication Aug 17, 2021.

doi: 10.21037/apm-21-2053

View this article at: https://dx.doi.org/10.21037/apm-21-2053 


\section{Introduction}

Inflammatory bowel disease (IBD) includes Crohn's disease (CD) and ulcerative colitis (UC). An epidemiology meta-analysis in China showed that the overall incidence of IBD in China is 1.74 [95\% confidence interval (CI): 1.08 to 2.40$] / 100,000$ people per year (1), which is increasing annually. The course of IBD presents the characteristics of alternating episodes of activity and remission, and incurability. In addition, long-term medication, regular outpatient follow-up, and repeated hospitalizations affect patient quality of life, and IBD patients may experience various psychological symptoms, such as anxiety, depression, and nervousness (2-4). As a part of comprehensive IBD therapy, although psychological intervention cannot cure clinical symptoms, it can effectively relieve symptoms, reduce negative emotions, and improve quality of life and happiness. Moreover, it can also reduce negative psychological effects on patients, and has a certain role in increasing their positive emotions $(5,6)$.

General well-being is a high-level psychological experience, an individual's overall evaluation of quality of life, which reflects social function and adaptive state. Positive psychological intervention uses positive emotions, personality advantages, and meaningful relationships to inject happiness, immersion, and meaning into lives in order to increase happiness (7). Psychological resilience refers to a person's ability or traits when faced with stress or adversity, enabling them to successfully adapt and develop. People with higher levels of psychological resilience tend to cope with negative events in a positive way, thus showing a higher quality of life and happiness (8). Mindfulness, as a positive psychological trait, is when an individual consciously maintains attention on the current internal or external experience. Without making any judgments, people can change their perception and tolerance to reduce psychological symptoms such as anxiety and depression, so as to reinforce healthy behaviors (such as compliance, diet, and exercise). Mindfulness exerts its psychological regulating effect during the current period, thereby improving the quality of life and happiness $(9,10)$. This study aimed to explore the correlation between mindfulness levels, psychological resilience, and overall well-being in patients with IBD, and the mediating effects of psychological resilience on mindfulness and happiness in patients with IBD. This was expected to provide a theoretical basis for exploring more suitable psychological intervention strategies for patients with IBD. We present the following article in accordance with the STROBE reporting checklist (available at https://dx.doi.org/10.21037/ apm-21-2053).

\section{Methods}

\section{Research participants}

This study investigated IBD patients who visited the Department of Gastroenterology, Jiangsu Provincial People's Hospital from February 2019 to February 2020 (participants were informed of and agreed to the study). The inclusion criteria were as follows: age $\geq 16$ years old; confirmed diagnosis of IBD; clear consciousness; willingness to cooperate; a certain ability of text interpretation. The exclusion criteria were as follows: the patient was unconscious or suffered from severe acute and chronic diseases such as heart failure, kidney failure, lung disease, liver disease, or mental illness (the metal illness was according to the history of psychiatric diseases diagnosed by the hospital). All procedures performed in this study involving human participants were in accordance with the Declaration of Helsinki (as revised in 2013). The study was approved by the Ethics Committee of the First Affiliated Hospital of Nanjing Medical University (Jiangsu Provincial People's Hospital) (NO.:2021-SR-405) and informed consent was taken from all the patients.

\section{Research methods}

(I) General Information Questionnaire: designed by researchers, including gender, age, education, source of medical expenses, course of disease, and so on. (II) The Connor-Davidson Resilience Scale (CD-RICS): the scale was originally compiled by Connor and Davidson, and translated and revised by Chinese academics (11). The scale has 25 items in 3 dimensions, including 13 resilience items, 8 strength items, and 4 optimistic items. They are scored on a 5-level Likert scale, with each item ranging from 0 to 4, meaning "never" to "almost", with a perfect score of 100. A higher score indicates better psychological resilience. The Cronbach $\alpha$ was 0.897 in this study. (III) Five Facet Mindfulness Questionnaire (FFMQ): FFMQ as revised by Meng et al. (12), with a total of 39 items in 5 dimensions, which are 8 items to observe, 8 items to describe, 8 items to act consciously, 8 items to no judgment, and 7 items to no act, adopting a Likert 5 -point scale. Each item ranges from 1 to 5 points, meaning "never" to "fully qualified". The higher the total FFMQ score, the higher the level of mindfulness. The Cronbach $\alpha$ was 
Table 1 General information

\begin{tabular}{|c|c|c|}
\hline $\begin{array}{l}\text { General } \\
\text { information }\end{array}$ & Subtypes & Number (\%) \\
\hline \multirow[t]{2}{*}{ Gender } & Male & $93(68.9)$ \\
\hline & Female & $42(31.1)$ \\
\hline Age & Mean \pm SD [range ] & $32.62 \pm 11.64[16-75]$ \\
\hline \multirow[t]{4}{*}{ Education } & Primary school student & $1(0.8)$ \\
\hline & $\begin{array}{l}\text { Junior middle school or high } \\
\text { school student }\end{array}$ & $40(29.6)$ \\
\hline & $\begin{array}{l}\text { Junior college student or } \\
\text { undergraduate }\end{array}$ & $87(64.4)$ \\
\hline & Postgraduate & $7(5.2)$ \\
\hline \multirow{2}{*}{$\begin{array}{l}\text { Medical } \\
\text { expenses }\end{array}$} & Medical insurance & $85(63.0)$ \\
\hline & Own pocket & $50(37.0)$ \\
\hline \multirow[t]{4}{*}{ Job } & Student & $24(17.8)$ \\
\hline & Worker & $69(51.1)$ \\
\hline & Unemployed & 39 (28.9) \\
\hline & Retired & $3(2.0)$ \\
\hline \multirow[t]{2}{*}{ Marital status } & Married & $111(83.0)$ \\
\hline & Unmarred & $24(17.0)$ \\
\hline \multirow{3}{*}{$\begin{array}{l}\text { Duration of } \\
\text { illness }\end{array}$} & $<1$ year & $6(4.0)$ \\
\hline & $1-5$ years & $64(47.4)$ \\
\hline & $>5$ years & $65(48.6)$ \\
\hline \multirow{3}{*}{$\begin{array}{l}\text { Degree of } \\
\text { illness }\end{array}$} & Remission or mild & $47(34.8)$ \\
\hline & Moderate & $80(59.3)$ \\
\hline & Severe & $8(5.9)$ \\
\hline \multirow[t]{5}{*}{ Medicine } & 5-aminosalicylic acid preparation & $47(34.8)$ \\
\hline & Hormones & $20(14.8)$ \\
\hline & Immunosuppressants & $28(20.7)$ \\
\hline & Biologics & $19(14.1)$ \\
\hline & Thalidomide & $21(15.6)$ \\
\hline
\end{tabular}

0.719 in this study. (IV) General Well-Being Schedule (GWB): GWB is a stereotyped test tool developed by the National Center for Health Statistics to evaluate subjects' statements of happiness. There are 33 entries in GWB, and 18 questions after revision in China. The higher the score, the stronger the happiness. National norms are $75.00 \pm 15.00$ points for males and $71.00 \pm 18.00$ points for females. Due to widely recognized and used in the evaluation system, these questionnaires and scales objectively can judge the relationship between mindfulness level, psychological elasticity and overall well-being.

\section{Research quality control}

Questionnaire design: Based on a large amount of literature and expert opinions, we selected a scientific and effective scale. Questionnaire compilation: the requirements were clarified and language was easy to understand. Preinvestigation: the questionnaire was refined based on the participant's feedback. The questionnaire was distributed by questionnaire star (www.wjx.cn) in 2 IBD WeChat groups (filled in by the participants themselves). The 2 investigators reviewed the filled-in information, and contacted the participant by phone to verify and make amendments if the initial response was not clear. The respondents whose questionnaires were completed in less than 15 minutes, where most of the answers were the same option, or determined to be filled in randomly after being contacted by phone were deemed invalid. A total of 184 questionnaires were issued and 159 were recovered, of which 135 were valid. The effective recovery rate was $73.3 \%$.

\section{Statistics}

Statistical analysis was performed using the software SPSS 21.0 (IBM Corp., Chicago, IL, USA) and Amos 24.0 (IBM Corp., Chicago, IL, USA). (I) Pearson correlation analysis was used to explore the relationship among mindfulness level, psychological resilience, and overall happiness. (II) The Amos 24.0 software was used to establish the structural equation model of the relationship among 3 variables: mindfulness level, psychological resilience, and overall happiness. (III) The bootstrap method was used to determine the mediating effect of psychological resilience between the level of mindfulness and overall well-being. The differences were considered significant if $\mathrm{P}<0.05$.

\section{Results}

\section{General information}

A total of 135 IBD patients participated in the study, of which 93 were male $(68.9 \%)$ and 42 were female $(31.1 \%)$ (Table 1). Their age was $16-75(32.62 \pm 11.64)$ years old. The ratios of education levels were $0.8 \%$ (primary school student), 29.6\% (junior middle school or high school 
Table 2 Correlation analysis of mindfulness level, psychological resilience, and overall well-being (r)

\begin{tabular}{|c|c|c|c|c|c|c|}
\hline Variable & Mindfulness & Psychological resilience & Resilience & Strength & Dimensions & Overall well-being \\
\hline Psychological resilience & $0.390^{*}$ & 1.000 & - & - & - & - \\
\hline Resilience & $0.366^{*}$ & $0.909^{*}$ & 1.000 & - & - & - \\
\hline Strength & $0.312^{*}$ & $0.891^{*}$ & $0.638^{*}$ & 1.000 & - & - \\
\hline Overall well-being & $0.391^{*}$ & $0.590^{*}$ & $0.560^{*}$ & $0.495^{\star}$ & $0.490^{*}$ & 1.000 \\
\hline
\end{tabular}

*, two-sided test, $\mathrm{P}<0.01 ;-$, blank.

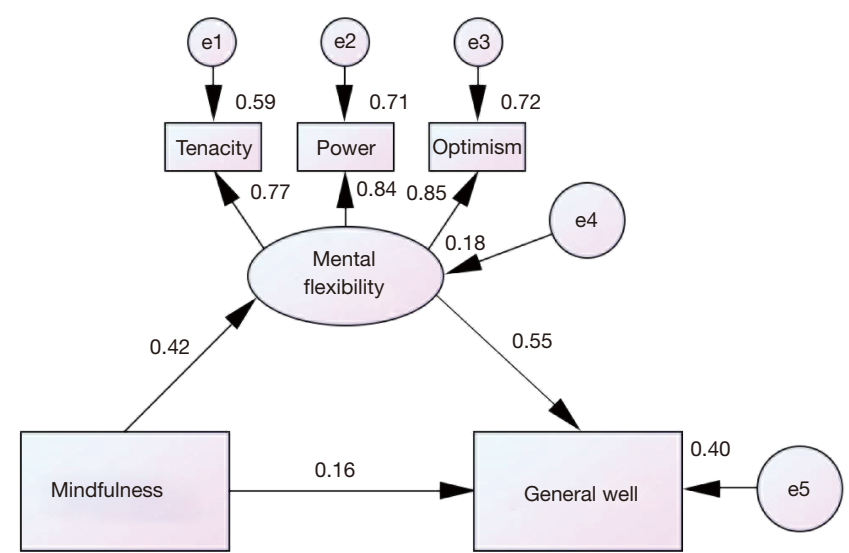

Figure 1 Structural equation model of mindfulness level, psychological resilience, and overall well-being.

student), $64.4 \%$ (junior college student or undergraduate), and $5.2 \%$ (postgraduate). A total of 85 participants (63\%) choose to use medical insurance to pay for medical expenses, and the others (37\%) paid themselves. Among them, there are 24 students, 69 office workers, 39 unemployed people, and 3 retirees, and only 24 were unmarried (17\%). In addition, 47 participants were in remission or had mild illness, and 80 and 8 had moderate and severe illness, respectively. Finally, a 5 -aminosalicylic acid preparation and hormones were used by 47 and 20 participants, respectively, while immunosuppressants, biologics, and thalidomide were used by 28,19 , and 21 participants, respectively.

\section{Current status of mindfulness, psychological resilience and overall well-being in IBD patients}

In this study, IBD patients had a mindfulness score of (119.95 \pm 8.64$)$, an observation score of $(22.13 \pm 4.65)$, a description score of $(24.83 \pm 4.12)$, a perceptually behavioral score of $(27.69 \pm 5.32)$, no judgment score of $(26.15 \pm 4.46)$, and no action score of $(19.33 \pm 5.14)$, respectively. In addition, the psychological resilience score was $(58.37 \pm 19.32)$, and the 3 dimensions of resilience, strength, and optimism were $(28.89 \pm 10.25),(20.77 \pm 8.39)$, and $(8.71 \pm 3.10)$, respectively. The overall well-being score was $(73.59 \pm 13.88)$. The male patients scored $(73.48 \pm 13.77)$, of which $52.7 \%$ were lower than the ordinary model; female patients scored $(73.81 \pm 14.27)$, of which $42.9 \%$ were lower than the ordinary model. Compared with the ordinary model, the differences were not statistically significant $(\mathrm{P}>0.05)$.

\section{Correlation analysis of mindfulness level, psychological resilience, and overall well-being}

Table 2 shows that the level of mindfulness was positively correlated with psychological resilience and its 3 dimensions (psychological resilience: $r=0.390$, resilience: $r=0.366$, strength: $\mathrm{r}=0.312$, optimism $\mathrm{r}=0.379 ; \mathrm{P}<0.01$ ). Meanwhile, overall well-being was positively correlated with psychological resilience and its 3 dimensions (psychological resilience: $r=0.590$, resilience: $r=0.560$, strength: $r=0.495$, optimism: $\mathrm{r}=0.490 ; \mathrm{P}<0.01)$. The level of mindfulness was positively correlated with overall well-being $(\mathrm{r}=0.391, \mathrm{P}<0.01)$.

\section{Mediating effect of psychological resilience between mindfulness level and overall well-being}

Next, we used the mindfulness level as the independent variable, overall well-being as the dependent variable, and psychological resilience and its 3 dimensions as the intermediate variables to construct a structural equation model (Figure 1). It was found that the path coefficients of the model were statistically significant $(\mathrm{P}<0.05)$ and 
Table 3 Structural equation model fit index

\begin{tabular}{lccccccccc}
\hline Fit index & CMIN/DF & RMSEA & GFI & AGFI & TLI & IFI & CFI & PGFI & NFI \\
\hline Test result & 1.947 & 0.084 & 0.977 & 0.913 & 0.965 & 0.986 & 0.986 & 0.260 & 0.972 \\
Fit standard & $1<\chi^{2} / \mathrm{df}<3$ & $<0.08$ & $>0.90$ & $>0.90$ & $>0.90$ & $>0.90$ & $>0.90$ & $>0.05$ & $>0.90$ \\
\hline
\end{tabular}

CMIN/DF, chi-square minimum degrees of freedom; RMSEA, root mean square error of approximation; GFI, goodness of fit index; AGFI, adjusted goodness of fit index; TLI, Tucker-Lewis index; IFI, incremental fit index; CFI, comparative fit index; PGFI, parsimonious goodness of fit index; NFI, normed fit index.

Table 4 Bootstrap analysis of the mediating effect of psychological resilience between mindfulness level and overall well-being

\begin{tabular}{lccccc}
\hline & \multirow{2}{*}{$\begin{array}{c}\text { Normalized } \\
\text { path coefficient }\end{array}$} & \multicolumn{3}{c}{$95 \% \mathrm{Cl}$} & \\
\cline { 3 - 4 } & $(\beta)$ & $\begin{array}{c}\text { Minimum } \\
\text { value }\end{array}$ & $\begin{array}{c}\text { Maximum } \\
\text { value }\end{array}$ & \\
\hline Direct effect $\left(c^{\prime}\right)$ & 0.159 & 0.008 & 0.312 & 0.035 \\
Indirect effect $(\mathrm{ab})$ & 0.232 & 0.138 & 0.332 & 0.001 \\
Total effect $(\mathrm{c})$ & 0.391 & 0.223 & 0.530 & 0.001
\end{tabular}

$\mathrm{Cl}$, confidence interval.

the fitting indexes were perfect, indicating that the model had been well constructed (Table 3). Meanwhile, we used the bootstrap method to detect the mediating effect of psychological resilience. Bootstrap repeat sampling was set to 2,000 and with $95 \% \mathrm{CI}$. The results showed that the direct effect was 0.159 , and the $95 \%$ CI was $(0.008$ to 0.312$)$ $(\mathrm{P}=0.035)$. In contrast, the indirect effect was 0.232 , and the $95 \%$ CI was $(0.138$ to 0.332$)(\mathrm{P}=0.001)$ (Table 4$)$. The study revealed that the direct and indirect effects of mindfulness level on overall well-being are statistically significant and $95 \%$ of the CIs did not include zero. Therefore, psychological resilience was shown to have a mediating effect on mindfulness level and overall well-being, and the mediating effect accounted for $59.3 \%$ of the total effect.

\section{Discussion}

\section{Mindfulness level, psychological resilience, and overall well-being in IBD patients}

Mindfulness emphasizes maintaining a present moment awareness of their thoughts, feelings, body sensations and surrounding environment. It also involves acceptance, meaning that people pay more attention to their thoughts and feelings without judgment (13). As a result, people can improve their psychological adjustment ability and adopt effective solution seeking skills. The mindfulness level of
IBD patients in this study was slightly lower than that of university students $(126.02 \pm 12.14)(14)$, but higher than that of IBD patients in Luo's study $(105.30 \pm 14.11)(15)$. There was no significant difference between mindfulness levels of patients with coronary heart disease (16) and IBD in this study. Both the observation and inaction scores are lower than 3 points, which indicates that IBD patients cannot maintain stable emotions and normal cognitive levels to cope with anxiety or depression when they experience recurrent illnesses or onset of symptoms that are prohibitive to participating in social activities. Therefore, mindfulnessbased stress reduction (MBSR) (17) or mindfulness-based cognitive therapy (MBCT) (18), which reduce the negative impact on individuals by improving their cognitive ability, can be applied to IBD patients.

This study revealed that IBD patients can experience decreased psychological resilience, especially in regards to optimism. These results are consistent with the findings of Kang (19). The main reason for this impact on psychological resilience is that IBD currently lacks a clear and effective cure, and treatment can only control or delay the onset of IBD. Patients with IBD are severely affected by the physical pain and inconvenience of life or social activities incurred by the disease. When patients experience frustration and dilemma, they lack a calm and positive attitude and adopt more negative coping strategies such as self-blame, retreat, and rationalization. These behaviors will compound their psychological stress, aggravate their condition, and create a vicious cycle. These conclusions suggest that in addition to paying attention to the patient's disease process, clinical nurses should proactively engage the patient's psychological resilience to improve their psychological resilience level and promote individuals to deal with stress and distress actively and effectively.

Overall well-being comes from the satisfaction of selfneeds and the fulfillment of ideals. As a comprehensive psychological index for evaluating the overall quality of life of an individual, it is a complex, multi-layered mental state 
formed by the interaction of psychological factors (including needs, cognition, and emotions) and external incentives. The overall well-being levels of male and female patients in this study were $52.7 \%$ and $42.9 \%$ lower than those in the ordinary model. The overall well-being of IBD patients is lower than normal, especially for males. The reasons are as follows: (I) intestinal symptoms and related complications at the onset of the disease cause physical pain. (II) The disease affects patients' daily lives and social activities, such as increased loneliness caused by inability to work and study and social alienation. (III) Increased financial burden caused by treatment costs. Due to differences in social division of labor and psychological factors, men may experience stress, which may be the reason why men's overall well-being below the ordinary model was proportionately higher than that of women. However, the overall average well-being was similar to the ordinary model. The reasons are as follows: (I) for mild patients, symptoms can be controlled through the efficacy of the drug. (II) The majority of patients surveyed in this study were young and middle-aged (mean age: $32.62 \pm 11.64$ ), they have received higher education due to educational reform; meanwhile, they had certain self-regulation ability and higher self-regulation subjective initiative. In addition, they had relatively stable economic sources and easier access to quality medical resources. (III) Other factors such as personal self-esteem (20), family care (21), social support (22), and so on can also increase overall patient well-being.

\section{The relationship between mindfulness level, psychological resilience, and overall well-being}

Pearson's correlation analysis showed that there was a positive correlation between mindfulness level, psychological resilience, and overall well-being, indicating that higher mindfulness level and better psychological resilience will engender higher overall happiness.

\section{Intermediary effect of psychological resilience between mindfulness level and overall well-being}

There was a correlation between mindfulness levels, psychological resilience, and overall well-being which met the basic criteria of intermediary effect. Intermediary effect analysis showed that mindfulness level and psychological resilience can positively predict overall well-being, supporting that mindfulness level and psychological resilience are predictors of overall well-being $(23,24)$.
Meanwhile, psychological resilience played an intermediary role between mindfulness level and overall well-being. This result indicates that mindfulness level not only directly affects overall well-being, but also indirectly regulates overall well-being through psychological resilience, which is a mediating variable. Psychological resilience is one of the mechanisms of the positive relationship between mindfulness level and overall well-being. Furthermore, bootstrap analysis further supported the existence of an intermediary effect in psychological resilience, which accounted for $59.3 \%$ of the total effect.

In conclusion, the mindfulness level, psychological resilience, and overall well-being of patients with IBD need to be improved. Mindfulness level and psychological resilience are predictors of overall well-being. Psychological resilience has a partial mediating effect between mindfulness level and overall well-being. This suggests that in the nursing process for IBD patients, clinical caregivers can directly increase mindfulness level through psychological intervention to improve happiness, or they can indirectly achieve the goal by enhancing psychological resilience.

\section{Acknowledgments}

Funding: This study was funded by Research on the precise nursing mode of chronic diseases based on comprehensive evaluation (2017-2022 Medical innovation team project provided by Scientific education project of Jiangsu Health Department[2017]1).

\section{Footnote}

Reporting Checklist: The authors have completed the STROBE reporting checklist. Available at https://dx.doi. org/10.21037/apm-21-2053

Data Sharing Statement: Available at https://dx.doi. org/10.21037/apm-21-2053

Conflicts of Interest: All authors have completed the ICMJE uniform disclosure form (available at https://dx.doi. org/10.21037/apm-21-2053). The authors have no conflicts of interest to declare.

Ethical Statement: The authors are accountable for all aspects of the work in ensuring that questions related to the accuracy or integrity of any part of the work are appropriately investigated and resolved. All procedures 
performed in this study involving human participants were in accordance with the Declaration of Helsinki (as revised in 2013). The study was approved by the Ethics Committee of the First Affiliated Hospital of Nanjing Medical University (Jiangsu Provincial People's Hospital) (NO.:2021-SR-405) and informed consent was taken from all the patients.

Open Access Statement: This is an Open Access article distributed in accordance with the Creative Commons Attribution-NonCommercial-NoDerivs 4.0 International License (CC BY-NC-ND 4.0), which permits the noncommercial replication and distribution of the article with the strict proviso that no changes or edits are made and the original work is properly cited (including links to both the formal publication through the relevant DOI and the license). See: https://creativecommons.org/licenses/by-nc$\mathrm{nd} / 4.0 /$.

\section{References}

1. Li X, Song P, Li J, et al. The Disease Burden and Clinical Characteristics of Inflammatory Bowel Disease in the Chinese Population: A Systematic Review and MetaAnalysis. Int J Environ Res Public Health 2017;14:238.

2. Jones JL, Nguyen GC, Benchimol EI, et al. The Impact of Inflammatory Bowel Disease in Canada 2018: Quality of Life. J Can Assoc Gastroenterol 2019;2:S42-S48.

3. Hood MM, Jedel S. Mindfulness-Based Interventions in Inflammatory Bowel Disease. Gastroenterol Clin North Am 2017;46:859-74.

4. Neuendorf R, Harding A, Stello N, et al. Depression and anxiety in patients with Inflammatory Bowel Disease: A systematic review. J Psychosom Res 2016;87:70-80.

5. Ludwig DS, Kabat-Zinn J. Mindfulness in medicine. JAMA 2008;300:1350-2.

6. Taft TH, Ballou S, Bedell A, et al. Psychological Considerations and Interventions in Inflammatory Bowel Disease Patient Care. Gastroenterol Clin North Am 2017;46:847-58.

7. Jaser SS, Whittemore R, Choi L, et al. Randomized Trial of a Positive Psychology Intervention for Adolescents With Type 1 Diabetes. J Pediatr Psychol 2019;44:620-9.

8. Zhang J, Zhang J, Liu S, et al. Correlation between mental resilience, anxiety, depression and subjective well-being of empty-nesters. Chin J Gerontology 2016;36:4083-5.

9. Xu W. Liu X. Mindfulness training enhances individual's well-being: A review. Chin Mental Health J 2013;27:197-202.
10. Wu L. The clinical value of mindfulness training in improving menopausal women's well-being and depressive symptoms. Chin J Gerontology 2016;36:4332-4.

11. Yu X, Zhang J. Factor analysis and psychometric evaluation of the Connor-Davidson Resilience Scale (CD-RISC) with Chinese people. Soc Behav Personal J 2007;35:19-30.

12. Meng Y, Mao K, Li C. Validation of a Short-Form Five Facet Mindfulness Questionnaire Instrument in China. Front Psychol 2020;10:3031.

13. Creswell JD. Mindfulness Interventions. Annu Rev Psychol 2017;68:491-516.

14. Teng S, Zhao J, Zhang X, et al. Chain mediating effect of mindfullness and sleep quality between negative life events and depression among college students. Chin J Behavioral Med Brain Science 2017;26:815-9.

15. Luo A. The intervention effect of mindfulness training on patients with inflammatory bowel disease and mood disorder. Central South University; 2013.

16. Wang J, Wang Q, Kuang C, et al. Studying on the Relationship Among Mindfulness, Stress Perception and Stress Handling of the Young and Middle -age with Coronary Artery Diseases. Chin Health Service Management 2107;34:458-60.

17. Jedel S, Hoffman A, Merriman P, et al. A randomized controlled trial of mindfulness-based stress reduction to prevent flare-up in patients with inactive ulcerative colitis. Digestion 2014;89:142-55.

18. Schoultz M, Atherton I, Watson A. Mindfulness-based cognitive therapy for inflammatory bowel disease patients: findings from an exploratory pilot randomised controlled trial. Trials 2015;16:379.

19. Kang N, Ma C, Wang Q, et al. Effectiveness of family intervention on resilience, anxiety and depression in patients with inflammatory bowel disease. J Nursing Science 2018;33:70-2.

20. Gao Y, Wang X, Zeng J, et al. Influence of nurses' sense of control on subjective well-being and mediating role of self-esteem. Chin Nursing Research 2017;31:2601-4.

21. Jing Y, Wan J, Shen J, et al. Analysis of influencing factors on subjective well-being in patients with stroke. J Nursing Administration 2018;18:622-6.

22. Ji Y, Luo J. The influence of leisure participation and social support on depression and happiness of the elderly. Chin J Gerontology 2019;39:1460-6.

23. de Vibe M, Solhaug I, Rosenvinge JH, et al. Six-year positive effects of a mindfulness-based intervention on mindfulness, coping and well-being in medical and 
psychology students; Results from a randomized controlled trial. PLoS One 2018;13:e0196053.

24. He F, Cao R, Feng Z, et al. The impacts of dispositional optimism and psychological resilience on the subjective well-being of burn patients: a structural equation modelling analysis. PLoS One 2013;8:e82939.

(English Language Editor: J. Jones)

Cite this article as: Wang M, Lu X, Liu M. The mediating effect of psychological resilience on the level of mindfulness and general well-being in patients with inflammatory bowel disease. Ann Palliat Med 2021;10(8):9215-9222. doi: 10.21037/apm-212053 